\title{
Simulation of the high-degree lithospheric field recovery for the Swarm constellation of satellites
}

\author{
S. Maus ${ }^{1 *}$, H. Lühr ${ }^{1}$, and M. Purucker ${ }^{2}$ \\ ${ }^{1}$ GeoForschungsZentrum, 14473 Potsdam, Germany \\ ${ }^{2}$ Raytheon ITSS at Geodynamics Branch, GSFC/NASA Greenbelt, MD 20771, USA
}

(Received January 15, 2005; Revised June 3, 2005; Accepted June 27, 2005; Online published April 14, 2006)

\begin{abstract}
A primary objective of the Swarm constellation mission is to resolve the lithospheric magnetic field with the best achievable accuracy in order to bridge the spectral gap between satellite and airborne/marine magnetic surveys. In a series of end-to-end simulations, the possibilities of high degree field recovery were investigated. The proposed constellation consists of a higher and a lower pair of satellites. It was soon found that a constellation as such does not yet guarantee improved high degree field recovery. Of crucial importance is the orbit constellation of the lower pair of satellites. If the lower satellites follow each other, as investigated in Constellation 1, the gain of a constellation turns out to be marginal, compared to a single satellite. For Constellation 2, the lower satellites were separated in the E/W direction. In this setup, one can use the instantaneous E/W magnetic field gradient between the satellites, as well as the N/S along track gradients. Incorporating this vector gradient information results in significantly improved field resolution. Indeed, the final simulation suggests that the envisaged Swarm constellation will enable the recovery of the lithospheric field to beyond spherical harmonic degree 130 .
\end{abstract}

Key words: Geomagnetic field, magnetic field modeling, crustal magnetic field.

\section{Introduction}

Mapping magnetic anomalies is one of the most successful and widely used techniques in geophysical exploration and geological mapping. During the past two decades, considerable efforts have been made to combine ground, airborne and marine magnetic surveys into continental scale compilations. However, due to the changing field from the Earth's core, individual surveys conducted at different years are offset against each other. Survey parameters such as flight altitude and direction can also differ. Arbitrarily bringing these disparate surveys to a common level distorts the long wavelengths. Consequently, the information in these compilations is limited to wavelengths of less than about $200 \mathrm{~km}$, depending on the individual input survey sizes and the availability of dedicated long range profiles.

Magnetic anomaly mapping gained a new dimension with the POGO (1967-1971) and Magsat (1979-1980) missions, which for the first time provided global magnetic anomaly maps (Langel and Hinze, 1998; Regan et al., 1975; Cohen and Achache, 1990; Arkani-Hamed et al., 1994; Ravat et al., 1995). Due to data quality issues and the short duration of the Magsat mission, the maps were limited to a resolution of about $1000 \mathrm{~km}$, corresponding to spherical harmonic (SH) degree 40 . After a pause of 20 years, measurements from three satellites with science quality mag-

*Now at CIRES, University of Colorado at Boulder and at NOAA's National Geophysical Data Center.

Copyright (c) The Society of Geomagnetism and Earth, Planetary and Space Sciences (SGEPSS); The Seismological Society of Japan; The Volcanological Society of Japan; The Geodetic Society of Japan; The Japanese Society for Planetary Sciences; TERRAPUB. netometers are now available for a period of more than five years. Ørsted (launched February 1999) and SAC-C (launched November 2000) have rather high orbital altitudes of around $700 \mathrm{~km}$, though, limiting the resolution of the crustal field to about degree 60. The CHAMP satellite, on the other hand, launched in July 2000 to an initial altitude of $450 \mathrm{~km}$, is providing high resolution data from which crustal field models to SH degree 90 have been derived (Maus et al., 2002, 2006). By the end of the CHAMP mission, at around 2008, the crustal field is expected to be resolved accurately to about $\mathrm{SH}$ degree 100 . Corresponding to a wavelength of $400 \mathrm{~km}$, this still leaves a considerable spectral gap between the CHAMP anomaly map and the continental scale compilations. The upcoming Swarm constellation mission (Friis-Christensen et al., 2006) provides a unique opportunity to close this gap.

Mapping the high degree lithospheric field is challenging due to the attenuation of shorter wavelength anomalies with altitude. The weak signal observed at satellite altitudes in the range of $300-450 \mathrm{~km}$ is easily masked by time varying external contributions to the geomagnetic field. The idea of the upcoming Swarm mission is to better distinguish spatial from temporal variations of the magnetic field by using a constellation of satellites. This provides a unique opportunity to map the high degree lithospheric field down to the longest wavelengths covered by the aeromagnetic/marine compilations.

Here, we describe a series of studies performed in the frame work of the Phase A Swarm end-to-end mission performance simulator (Olsen et al., 2006) to investigate the possibilities and limitations of high-degree lithospheric field mapping, and to find optimum orbital constellations 
Table 1. Virtual satellites in Constellations 1 and 2.

\begin{tabular}{lcccc}
\hline Name & Renamed to & Altitude & Inclination & Comment \\
\hline C1-S1 & & $550 \mathrm{~km}$ & $86.0^{\circ}$ & \\
C1-S2 & & $550 \mathrm{~km}$ & $86.0^{\circ}$ & following C1-S1 with 180 separation \\
C1-S3 & & $850-250 \mathrm{~km}$ & $85.4^{\circ}$ & \\
C1-S4 & & $850-250 \mathrm{~km}$ & $85.4^{\circ}$ & following C1-S3 with 1000 km separation \\
C1-S5 & & $450-250 \mathrm{~km}$ & $85.4^{\circ}$ & following C1-S3 with 3000 km separation \\
C2-S1 & Swarm C & $550 \mathrm{~km}$ & $87.3^{\circ}$ & \\
C2-S2 & & $550 \mathrm{~km}$ & $87.3^{\circ}$ & following C2-S1 with $180^{\circ}$ separation \\
C2-S3 & & $550 \mathrm{~km}$ & displaced E/W to C2-S1 by 12 \\
C2-S4 & Swarm A & $450-250 \mathrm{~km}$ & $86.8^{\circ}$ & \\
C2-S5 & & $450-250 \mathrm{~km}$ & $86.8^{\circ}$ & displaced E/W to C2-S4 by about $1.5^{\circ}$ \\
C2-S6 & & $450-250 \mathrm{~km}$ & $86.8^{\circ}$ & displaced E/W to C2-S4 by $12^{\circ}$ \\
C2-S7 & Swarm B & $450-250 \mathrm{~km}$ & $86.8^{\circ}$ & as C2-S5, but strict $1.5^{\circ}$ separation \\
\hline
\end{tabular}

for this purpose, in the presence of the complete set of magnetic field contributions. These simulated contributions ranging from the Earth's core to the magnetosphere are described in detail in Olsen et al. (2006). However, the production of the synthetic input data was not part of the study presented here. The input models were chosen by a larger committee, and the synthetic data were produced by a separate team. These data were then downloaded from a central FTP site by all participating science teams for their respective field recovery simulations (see companion papers in this special issue).

Two orbital constellations were investigated. Both constellations consisted of several satellites in higher orbits at about $550 \mathrm{~km}$ altitude and several lower satellites, descending from $450 \mathrm{~km}$ to $250 \mathrm{~km}$ in altitude over the mission duration of 4 years. They are summarized in Table 1 . The higher and lower satellites commence with orbital planes at almost equal local times (being launched by a single vehicle). The orbital planes of the higher satellites then slowly drift apart from the orbital planes of the lower ones. The main difference between constellations 1 and 2 is that the lower satellites in the first constellation (C1-S3, C1-S4 and C1-S5) followed each other, like the GRACE gravity satellites (Tapley et al., 2004), while in Constellation 2 the lower satellites were displaced in the $\mathrm{E} / \mathrm{W}$ direction. For Constellation 2, different spatial separations were investigated, namely C2-S4/C2-S5 with an E/W separation of $1.5^{\circ}$ at the equator, and C2-S4/C2-S6 separated by $11.25^{\circ}$. Since the $\mathrm{C} 2-\mathrm{S} 4 / \mathrm{C} 2-\mathrm{S} 5$ pair did not maintain the $1.5^{\circ}$ longitudinal separation very well over the course of the simulated mission, a satellite C2-S7 with an actively maintained longitudinal separation of $1.5^{\circ}$ to $\mathrm{C} 2-\mathrm{S} 4$ was introduced for the final high-degree lithospheric field recovery study.

In the first part (Section 2) we recovered the lithospheric field from "clean" data that only contained the lithospheric signal. This was an important initial step for testing the reliability of the algorithms involved. The questions to be addressed included an assessment of the consistency of the synthetic data, a check of the ability of the inversion algorithm to recover the SH coefficients, and an investigation of the role of the polar gap. The synthetic magnetic data contained features of scale sizes much smaller than have ever been recovered from satellites. Therefore, it was initially not clear to what extent they could be recovered from the synthetic data in this inversion. In the second part of this study (Section 3) we used the same orbital constellation (Constellation 1) but the data included all source contributions. After it became clear that little was gained from two spacecraft closely following each other in the same orbit, Constellation 2 was designed with the two lower spacecraft flying side-by-side, separated in the $\mathrm{E} / \mathrm{W}$ direction and crossing the equator approximately at the same time. In addition, synthetic instrument noise was added to the data. Using a specially developed gradient method, studies based on Constellation 2 data showed a significantly improved field recovery. The study also yielded the intuitive rule that the spacecraft separation must be smaller than the wavelength of the smallest scales to be recovered in order to be able to measure meaningful gradients.

\section{Inversion of Lithosphere-only Data}

In an initial study, the accuracy of the synthetic data set and the feasibility of the inversion approach were tested. Only the lithospheric field contribution was used in this initial investigation. The synthetic data were derived from a static field model from SH degree 14 to 120 (Olsen et al., 2006). At degrees 1-13 the core field dominates over the lithospheric magnetic field. Model coefficients above $n=110$ had been tapered to reach zero at $n=120$. The tapering was meant to prevent the presumed ringing of a sharp cut-off. However, it was later recognized that it would be more realistic to continue the synthetic lithospheric field to a much higher SH degree without tapering, as implemented in the high-degree experiment of Constellation 2. The individual field values were truncated at a resolution of $0.01 \mathrm{nT}$. This reflects the approximately achievable degree of quantisation of modern instruments, confirmed by the observed high frequency noise level of the CHAMP vector magnetometer. The sampling rate of the data set was 1 per minute, which, at a satellite speed of about $8 \mathrm{~km} / \mathrm{s}$, corresponds to a distance of $480 \mathrm{~km}$ between the samples. For the real situation with a time varying background field, a spacing wider than the wavelengths to be recovered can generate serious aliasing problems. The sampling rate was therefore reduced 


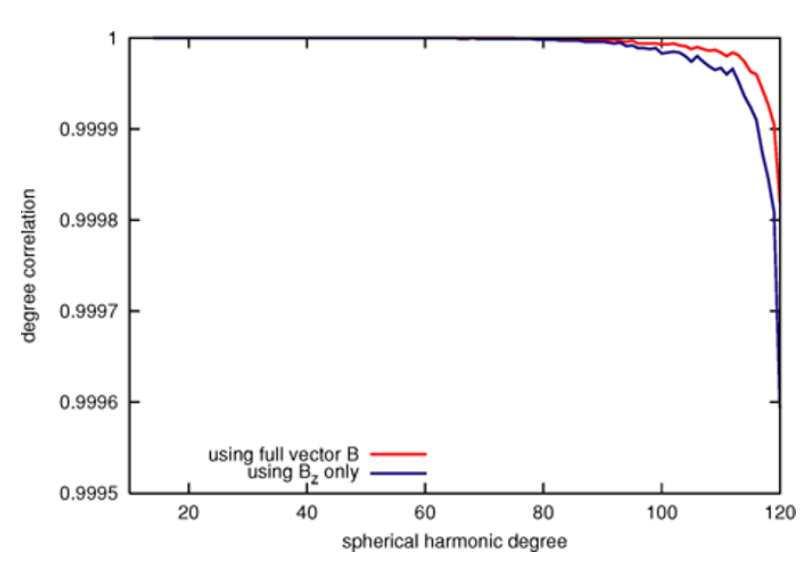

Fig. 1. Degree correlation between the input and recovered models for lithosphere-only data.

to 5 seconds for the later tests. However, for the initial test, with a static input field, a higher resolution was achieved by the repetition of orbits.

The mission simulations were carried out assuming a mission period of 1998 to 2001, one solar cycle prior to the planned Swarm mission, the reason being that auxilliary observatory data and magnetic and solar indices were available for this period. Due to the strong attenuation of the high degree lithospheric field with altitude, the later part of the mission was most favorable for this study. For the inversions of lithosphere-only data, we therefore used only the last 400 days, from day 330 to 730 in MJD2000 (November 2000-December 2001). The selected data were from one of the low satellites, C1-S3. This spacecraft traversed the altitude range of $400 \mathrm{~km}$ down to $250 \mathrm{~km}$ during the time span considered.

A quantity which was of particular concern for the investigations performed here is the size of the polar gap. Due to the inclination of the $\mathrm{C} 1-\mathrm{S} 3$ spacecraft of $85.4^{\circ}$, a circular area with a diameter of about $1000 \mathrm{~km}$ was left unsampled. This is significantly larger than the $333 \mathrm{~km}$ wavelength of a degree 120 spherical harmonic.
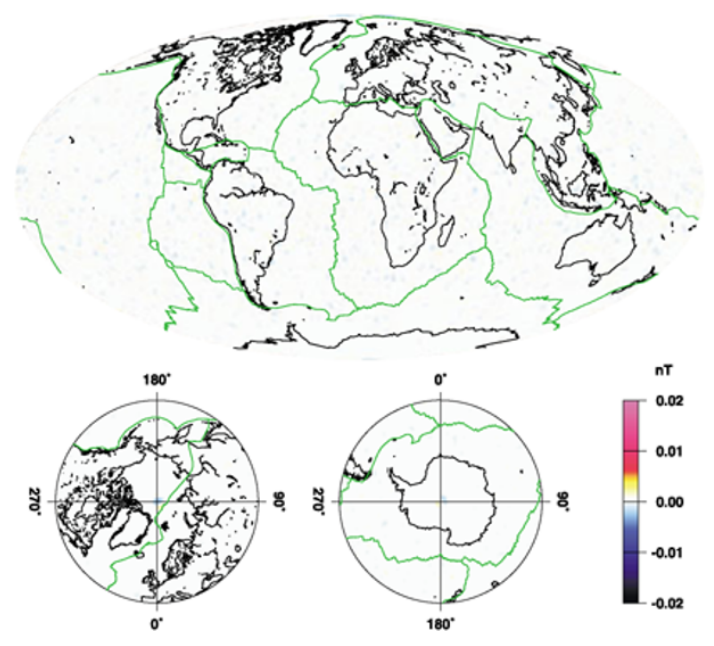

\subsection{Inversion approach}

The basic method which was employed here is the leastsquares algorithm for retrieving $\mathrm{SH}$ coefficients from vector measurements well distributed over a sphere (Maus et al., 2006). Due to the sampling pattern of a satellite in a near-polar orbit the density of measurement points is much higher at polar latitudes than close to the equator. This disparity is compensated by down-weighting the high-latitude samples. To achieve this, the data were counted in equal area bins and downweighted by their number density to obtain an equal area distribution on the sphere. After this treatment, all areas (except for the polar gap) contributed equally to the global solution. Since we are dealing with noise-free data, no weighting was used in the inversions. The applied standard inversion algorithm finds a solution by minimising the root mean square of the residuals.

In a first run we used the full vector information (three components). From a mathematical point of view, the full vector contains quite a bit of redundant information and it should be sufficient to use either the vertical or the two horizontal components to invert for an internal poloidal field in the possible presence of toroidal fields. To test this redundancy, all runs were repeated using only the vertical component.

\subsection{Recovered field from lithosphere-only data}

Figure 1 shows the degree correlation (Langel and Hinze, 1998) between the (true) input SH coefficients and the retrieved ones. According to this test, they are virtually identical. The correlation only drops to below unity when the tapering beyond degrees 110 sets in, where we obviously run out of signal. The full vector solution gives a slightly better result, probably because the horizontal components help to constrain the field in the polar gap.

A direct way of visualising the fit between input and output fields is to plot the field differences on a global map. Figure 2 (left) shows the results from the full vector solution for the vertically downwards component $\mathrm{B}_{z}$. Residuals are very small-well below the quantisation step size of $0.01 \mathrm{nT}$, even over the polar gaps. For the case of the solution using only the vertical component input data (cf. Fig. 2,

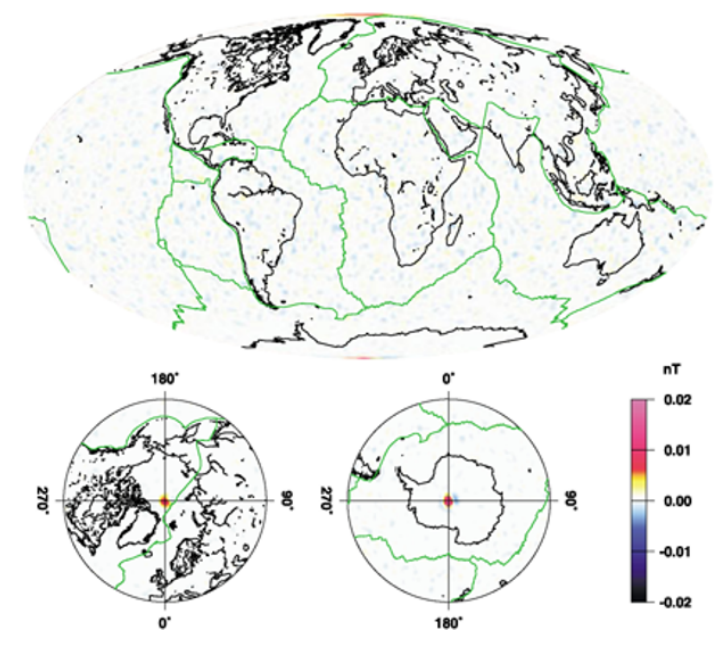

Fig. 2. Global distribution at $400 \mathrm{~km}$ altitude of the difference in $\mathrm{B}_{z}$ between the recovered and input model fields using as input to the inversion all vector components (left) and the vertical component only (right). 

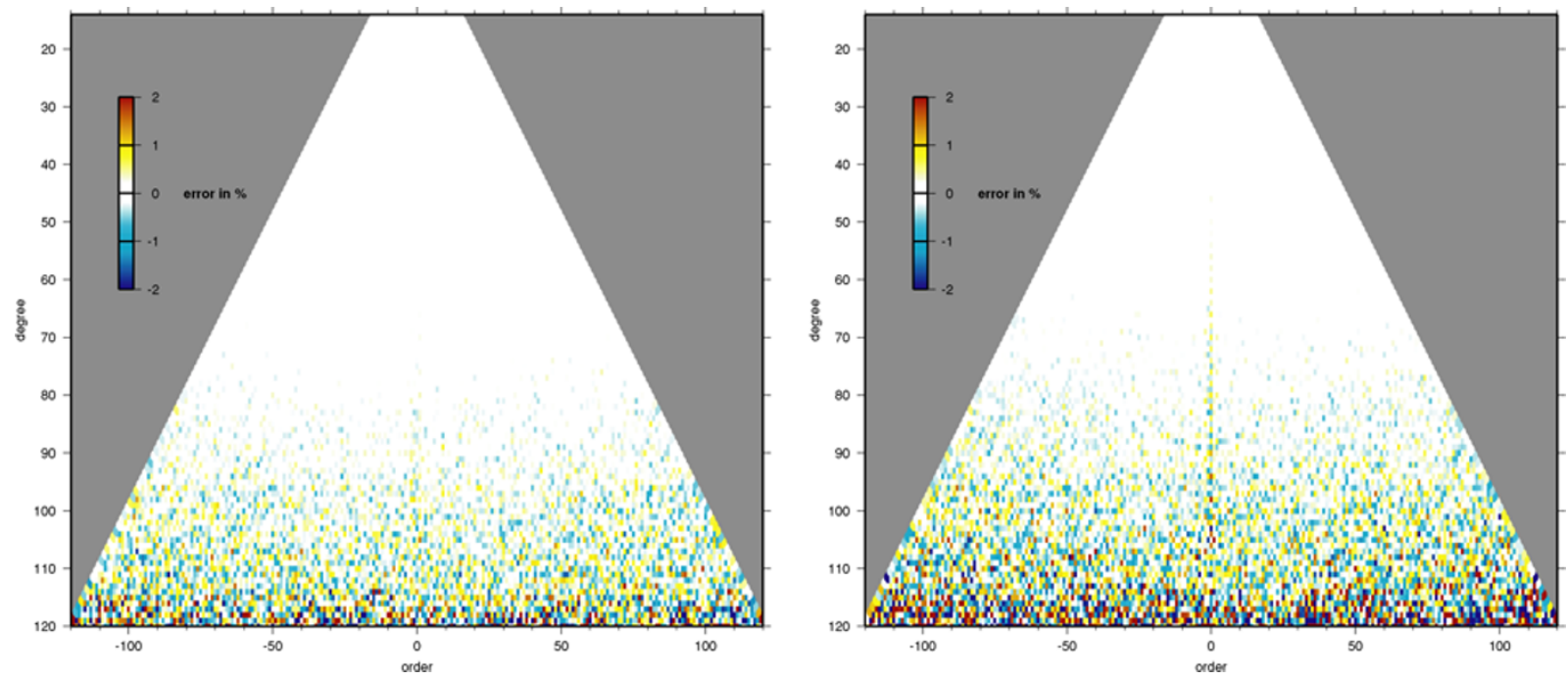

Fig. 3. Sensitivity matrix showing the difference between recovered and the input model SH coefficients for lithosphere-only data as relative errors, using all vector components (left) and only the vertical component (right).

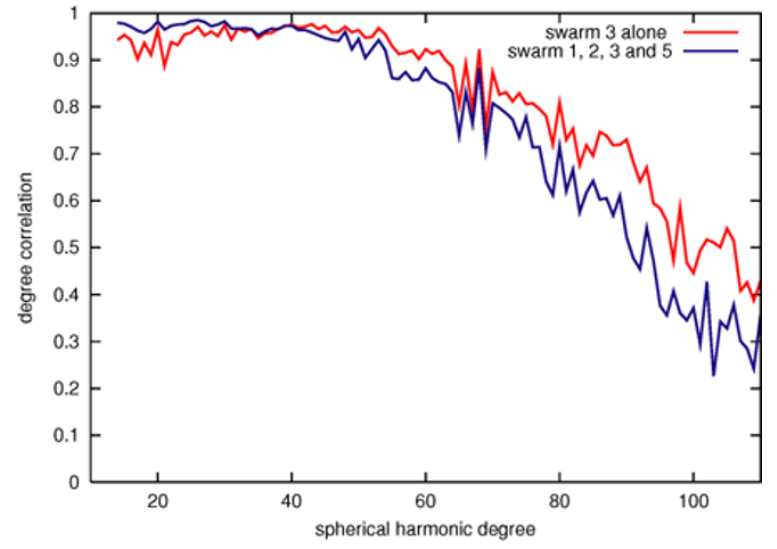

Fig. 4. The degree correlation between the input and the retrieved lithospheric magnetic field model for a single satellite (C1-S3) and a formation (C1-S1, C1-S2, C1-S3, and C1-S5) approach in Constellation 1.

right) the differences are slightly larger, but still insignificant and evenly distributed. As an interesting feature, for the latter case the polar gap becomes visible in both hemispheres. The horizontal components of the field observed close to the gap obviously help to constrain the field within the gap. Using only the vertical component, we lose this extra information with the effect that the field in the polar gap is not resolved.

In a third evaluation, the $\mathrm{SH}$ coefficients of the original and the recovered model are directly compared. The differences between the coefficients are the errors of the recovery. They can be displayed as a colour-coded sensitivity matrix with degree $n$ versus order $m$. Figure 3 (left) shows the percentage error for the full vector solution. Here the deviations are generally well below $1 \%$, except for the degrees just below $n=120$ where tapering becomes effective. Slightly larger errors are encountered when only the vertical component is used in the inversion (cf. Fig. 3, right). Worth noting is the distinct feature for coefficients with or$\operatorname{der} m=0$. These zonal coefficients are particularly sensi- tive to the field at the very highest latitudes (see figure 3.5.2 of Backus et al. (1996) for the behaviour with latitude of the Associated Legendre Functions). The somewhat enhanced errors of these zonal terms are caused by the polar gap. The polar gap obviously requires special attention in the Swarm orbital configuration.

\subsection{Discussion of results from the lithosphere-only in- version}

The comparison between the synthetic input data and the retrieved magnetic field model reveals almost complete recovery in the case of clean data. Indeed, the agreement is much better than the maximum error predicted for the linear 3D grid interpolation algorithm used to synthesise the lithospheric field (Olsen et al., 2006). Such an algorithm was used in the data synthesis in order to avoid the excessive computation times required for point by point SH transforms.

Several conclusions can be drawn from the results obtained. The truncation of the generated magnetic field data to a resolution of $0.01 \mathrm{nT}$, which is achievable with state-ofthe-art instrumentation, causes no limitation in the recovery of lithospheric signatures up to degree $n=120$. Also, the rather coarse sampling of 1-per-min had no negative effect on the solution. However, this conclusion may not hold up in a realistic setup with time-varying external fields. In the following simulations, where the complete field is represented in the input data, including its temporal variations, we used a denser sampling of one reading per $5 \mathrm{~s}$.

A feature of the input data which affects all of the results presented, is the tapering of the spectrum for degrees beyond $n=110$. No reliable recovery of the magnetic signatures could be obtained at this short wavelength end due to the tapering of the input signal. This effect is rather prominent in the sensitivity matrix (cf. Fig. 3). Errors above $2 \%$ are found only in the tapered degrees beyond $n=110$. From these findings it was recommended that for the following simulations the input lithospheric model should not be tapered and should be extended to higher degrees. Note that the input models for producing the synthetic data were 
not chosen by the authors of this study, but by the whole Swarm science team.

As expected, employing the full magnetic field vector for the inversion gives somewhat better results than determining the SH coefficients from only vertical component data. Quite prominent is the difference in the response to the polar gap. Even though it is rather large (about $1000 \mathrm{~km}$ in diameter) it was hardly discernible when the full vector data were used. When recovering the model from the vertical component only, larger differences showed up in both the global map (Fig. 2, right) and the spectral sensitivity matrix (Fig. 3, right). The enhanced errors for coefficients with $m=0$ are a manifestation of the polar gap.

When retrieving magnetic field models from real satellite measurements one usually uses only the field magnitude (or downweights the components perpendicular to the main field) in the polar regions, since the perpendicular (horizontal) components are strongly affected by ionospheric currents. In this respect, our solution using only the vertical component may describe real conditions better than the one using the full vector. At high latitudes the field direction is almost vertical over a fairly large range. We may conclude that a polar gap of $1000 \mathrm{~km}$ in diameter will have adverse effects on high resolution magnetic field modelling. To make use of the full performance of the Swarm mission will require a significantly smaller sized polar gap.

\section{Lithospheric Field Recovery from Constella- tion 1 Data}

For the second investigation, all source terms of the synthetic data set of Constellation 1 were added. These were the core field, including secular variation, crustal field, ionospheric, magnetospheric, Earth induced, and toroidal external fields. Here we made use of the 5-second data which provide a sufficiently dense sampling of the highdegree lithospheric signals. The purpose of this study was to show how well the lithospheric field could be recovered from the complete, but instrument-noise-free, data set when one or more satellites were included in the inversion.

In Section 2 we demonstrated that the lithospheric field could be reconstructed almost perfectly, even with a single satellite, if the synthetic input data consist only of the lithospheric source term. The multi-satellite approach thus had to be optimised toward an efficient separation of the other source terms. Since the external field geometry can be quite complex, four globally distributed satellites are not expected to provide sufficient extra information to provide a unique representation of all of the contributing source fields at a given point in time for the whole globe. We therefore reduced the size of the spatial area considered to a diameter of about $3000 \mathrm{~km}$, assuming that four satellites in a smaller area can provide a local description of the complete external field structure sufficiently well. The idea is that external fields on the night side are predominantly large scale and therefore can be identified using a small number of satellites measuring simultaneously in a small region. This approach resulted in the following data selection criteria:

- Only night-time (19:00 LT to 05:00 LT) passes were considered in order to minimise the effect of iono- spheric currents.

- Only time intervals with night-side equator crossings of all four satellites within a sector of $20^{\circ}$ in longitude were taken into account.

The second condition was met during the first 200 days of the simulated mission and once again for 400 days, roughly centred about the fourth year. We used both intervals (see Olsen et al. (2006) for the orbit development). It was not required that the satellites passed the region of interest simultaneously. We allowed for a time difference of up to one hour for tracks to be considered in a joint inversion. Variations taking place on this time scale were considered to be small and randomly distributed. This rather generous condition allowed all four satellites to contribute to the solution on almost every orbit during the periods considered. While the main field was assumed to be known to degree 13 and was subtracted from the synthetic data, the constellation approach was used to characterise the main features of the external field and its induction effect. Only the lowdegree terms of the disturbance field were determined. This was assumed to be sufficient for a local solution. On an orbit-by-orbit basis the following parameters were solved for:

- External magnetic dipole, aligned with the main dipole, plus its time derivative (2 parameters)

- An orthogonal external dipole in the orbital plane (1 parameter)

- The induced counterparts of these two components and the time derivative of the internal dipole ( 3 parameters)

- The toroidal field contribution in the geomagnetic longitudinal $\varphi$ direction (1 parameter)

- External magnetic quadrupole, axial component (1 parameter)

- Orthogonal quadrupole in orbital plane (2 parameters)

- The induced counterparts of the quadrupoles (3 parameters)

In a test run, we verified that these parameters are almost uncorrelated and therefore could be determined reliably on a track-by-track basis. The magnetic field components resulting from this joint multi-satellite inversion were considered to be long wavelength noise which was subtracted in order to isolate the lithospheric magnetic signal. This approach is also referred to as track-by-track filtering.

\subsection{Inversion result for Constellation 1 data}

In a first run we tested the algorithm by applying it to a single satellite, the low flying C1-S3. The SH coefficients were solved for from degrees 1 to 110 . Degrees 1-13 are dominated by the core magnetic field and were therefore discarded. The same inversion algorithm was then used in a multi-satellite inversion. As expected, the agreement with the original lithospheric field signature was not as good as in the case of the inversion of clean data. Figure 4 shows the degree correlation between the retrieved and input highdegree coefficients of the lithospheric field. An interesting result is that the single-satellite solution gave a better correlation over large parts of the spectrum than the multisatellite approach. This contrasted with the widespread belief that multiple satellites per se are a guarantee of bet- 

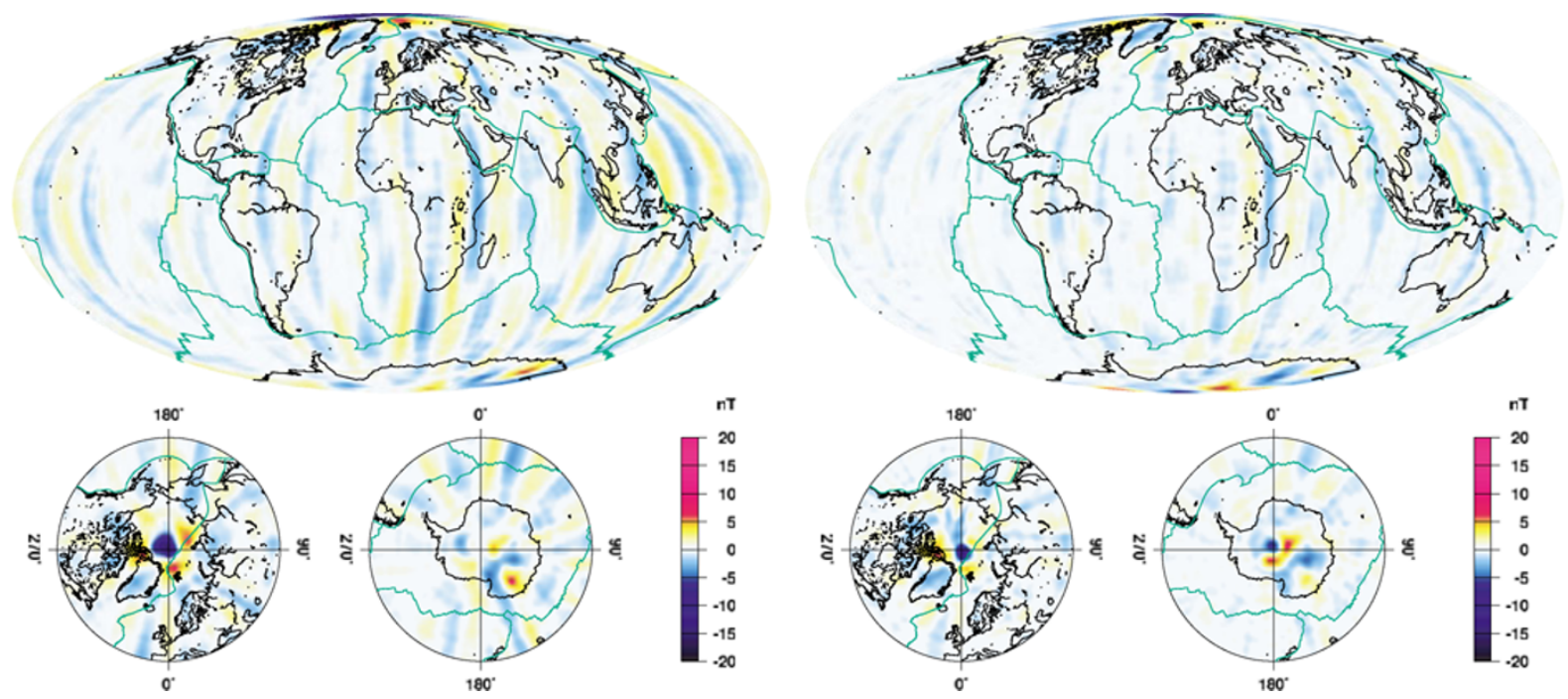

Fig. 5. Global distribution at $400 \mathrm{~km}$ altitude of $B_{z}$ residuals between input and derived model for the single satellite (left) and multi-satellite solution (right) in Constellation 1. The low-degree sectorial stripes are quite prominent.
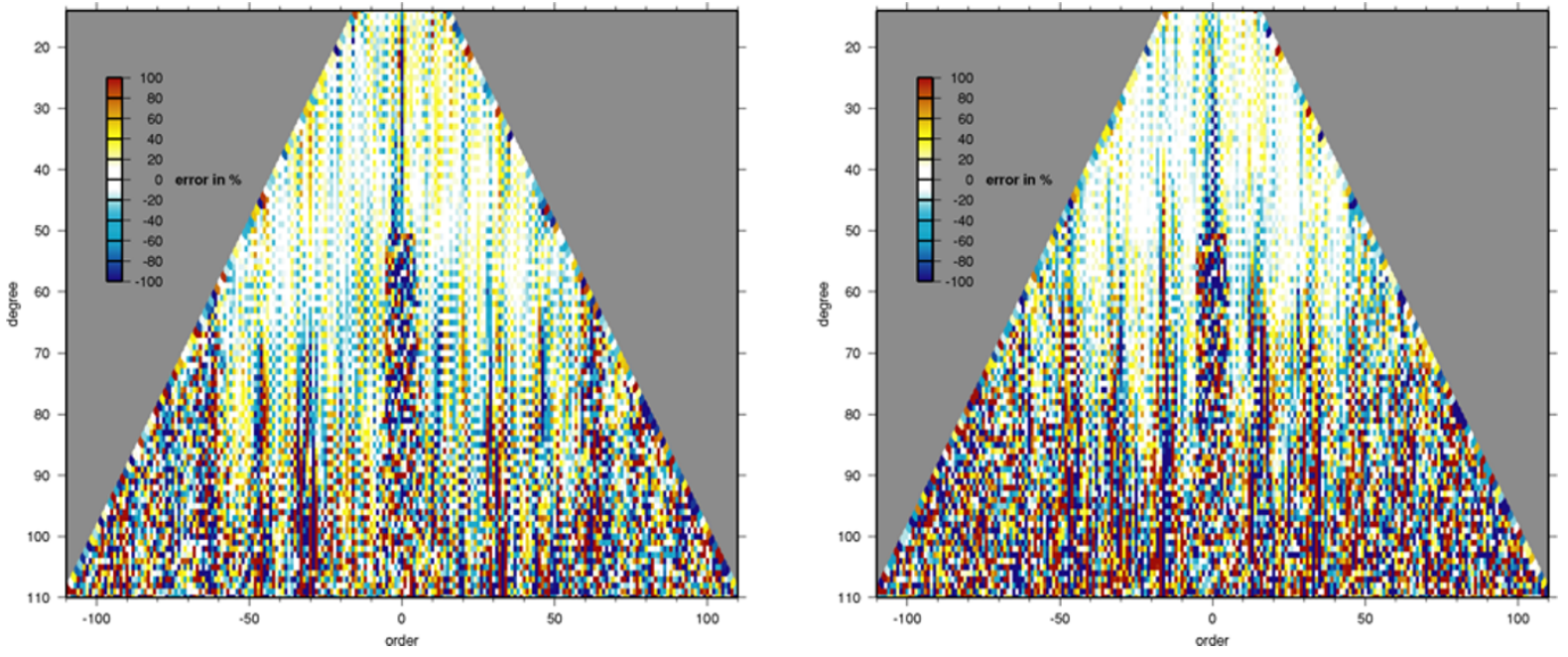

Fig. 6. Sensitivity matrix showing the difference between the input and the derived SH coefficients as relative errors for the single satellite (left) and multi-satellite results (right) in Constellation 1.

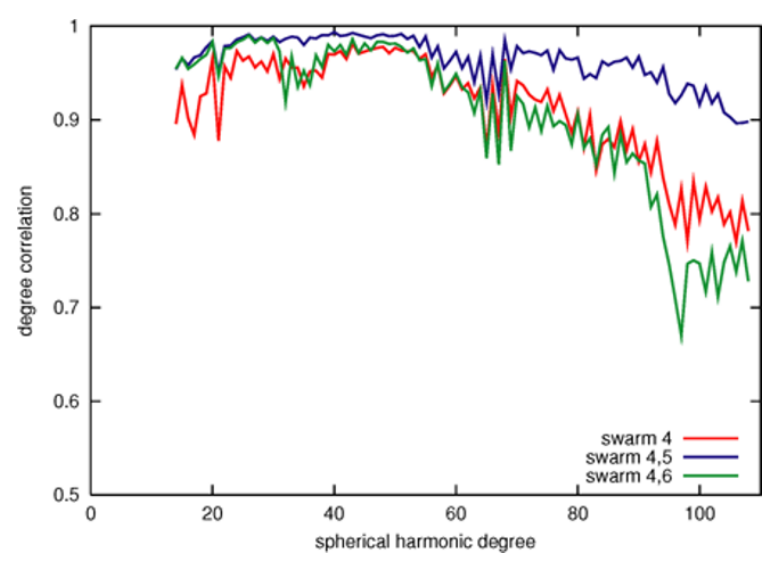

Fig. 7. Degree correlation between the input model and the retrieved lithospheric field model, separately for a single satellite solution (C2-S4) and for pairs of satellites (C2-S4/C2-S5 and C2-S4/C2-S6) in Constellation 2. ter field modelling results. It was agreed prior to the study (Olsen et al., 2006) that recovered models would be considered to be in agreement with the original input model for all degrees at which the degree correlation was above 0.7 . This threshold of 0.7 is encountered at degrees around 90 in the case of C1-S3 alone and slightly less than 80 for the foursatellite solution. The degree of field recovery with C1-S3 is remarkably high and goes beyond the resolution obtained so far by the CHAMP magnetic field measurements. This is due to the (implicitly assumed) superior measurement accuracy of the Swarm satellites and due to the fact that data at altitudes down to $300 \mathrm{~km}$, which is lower than the present CHAMP altitude, have been used.

Another way of visualising the fit between the original and the recovered model is to plot the local differences on a global map. Figure 5 shows the global distribution of the residuals for the single-satellite and the formation (multisatellite) solutions, respectively. The dominating features are sectorial stripes of residuals with alternating sign and 
amplitudes of up to $5 \mathrm{nT}$. Their spatial scales correspond to the lower truncation degree of $n=14$, indicating that the longest wavelengths are affected strongest. These stripes probably show the genuine lithospheric signal lost in the track-by-track filtering. In the case of the constellation solution, the stripes are much weaker, indicating that a smaller portion of the genuine lithospheric signal was filtered out. Spots of strong residuals appear in polar regions and, in particular, at the magnetic poles.

In the third evaluation, the retrieved $\mathrm{SH}$ coefficients are directly compared with the input model. Figure 6 shows the percentage errors for the single and multi-satellite solution respectively. Errors are colour-coded in the sensitivity matrix containing degree $n$ versus order $m$. The matrices show some interesting structures. At low degrees up to about $n=50$ the errors are fairly low. For higher degrees they increase significantly. There are prominent error levels at certain values of the order $m$. They come at multiples of $m=15$, which indicates that they are caused by mission periods in which the satellites went into exact-repeatorbits. These exact-repeat-orbits are disadvantageous, since the field is then repeatedly sampled at the same locations, while other locations remain unsurveyed. They occur at certain altitudes during the orbital decay of the satellite. In practice, they can be avoided by actively lowering a satellite through these resonant altitudes. It was not done in the simulation, but is strongly recommended for the real mission. Most pronounced in the sensitivity matrix is the peak at $m=0$. Again, this is due to the polar gap in the measurements.

\subsection{Discussion of the inversion of the Constellation 1 data}

In summary, the second simulation was based on noisefree data, but all magnetic field sources included in the synthetic data set (including the toroidal field) were considered in the inversion. Non-lithospheric field contributions were removed by track-by-track filtering. As expected, the results were not as good as in the first case (Section 2) where only the lithospheric field part was considered. The resolution achieved with the single satellite, obtaining correlations above 0.7 for $\mathrm{SH}$ degrees up to 90 , is very satisfying and can be interpreted as a confirmation of the efficacy of the filter used.

In the case of the multi-satellite inversion, a common solution, fitting all four satellites simultaneously, was sought. Figure 4 shows that the constellation solution is better for degrees up to 35 . In this wavelength range, the filter obviously removes genuine crustal signal when applied to data from a single satellite. For higher degrees, the filter fails to completely remove the non-lithospheric field. A stronger filter (co-estimating more parameters) could have improved the multi-satellite solution. However, this would not have led to an improvement at high degrees over the singlesatellite solution, as is apparent from the tests described below.

The efficiency of the constellation at long wavelengths is supported by the distribution of the residuals in Figure 5. The amplitude of the dominating sectorial stripes is greatly reduced in the constellation approach. The problem of single-track line levelling uncertainty inherent to track-by-track filtering can be effectively mitigated in the case of multi-satellite solutions. The optimal spacing of the spacecraft for this purpose is investigated below. It is worth noting that none of the prominent crustal magnetic features (Bangui, Kursk, Kiruna anomalies) are visible on these residual maps. The crustal field itself is obviously well recovered, but contaminated by additional signal in the long wavelength range.

On the other hand, Constellation 1 did not yield an improved high-degree lithospheric field recovery over a single satellite solution. The main reason was that the two lower satellites, closely following each other, simply replicated each other's measurements, while the higher satellites were too high in altitude to constrain the short scale field.

\section{Lithospheric Field Recovery from Constella- tion 2}

Inspired by the results of the previous investigation, it was decided to simulate the lower satellites flying sideby-side, rather than following each other, for Constellation 2. The following section investigates the advantages of the new constellation. In addition, a more realistic model of magnetospheric and induced contributions was used and realistic instrument noise was added to the synthetic data (Olsen et al., 2006).

\subsection{Data selection and inversion approach}

For this third study the synthetic data generated for Constellation 2 (Olsen et al., 2006) were used. We made full use of the 5-sec vector data. The dense sampling was needed for the recovery of the high-degree lithospheric anomalies. All magnetic source terms in the data set had been summed. In this case, realistic instrument noise was also included. There was one exception from the complete set of source terms. The synthetic toroidal field components were not considered because the toroidal field model was derived from Ørsted observations only $(650-850 \mathrm{~km})$. Their extrapolation to the lower Swarm altitudes turned out to be unstable and the result had no resemblance to real CHAMP observations at this altitude.

Data were only used for periods when ionospheric currents were known to be weak. Commonly used criteria are a small activity index, $K p \leq 2$ and the hours of the late night, 22 to 05 local time, when the ionospheric conductivity is low. Data for the whole simulated mission time of 61 months were considered in the selection, according to the above mentioned criteria, and treated in one inversion run for the retrieval of the lithospheric field.

A major task for recovering the rather weak lithospheric signal was the elimination of all the other source contributions. As a first step, a main field model was subtracted. Secular variation up to degree 13 was assumed to change linearly with time. The true input synthetic main field actually had a higher order cubic spline secular variation. Thus, our linear approximation deliberately introduced a realistic main field model error into the simulation.

Contributions from the external sources were again determined on a track-by-track basis. This time they were resolved up to degree 3 and order 1. Simultaneously, their induced counter-parts were estimated. If several satellites of the constellation were considered in an inversion run, all 

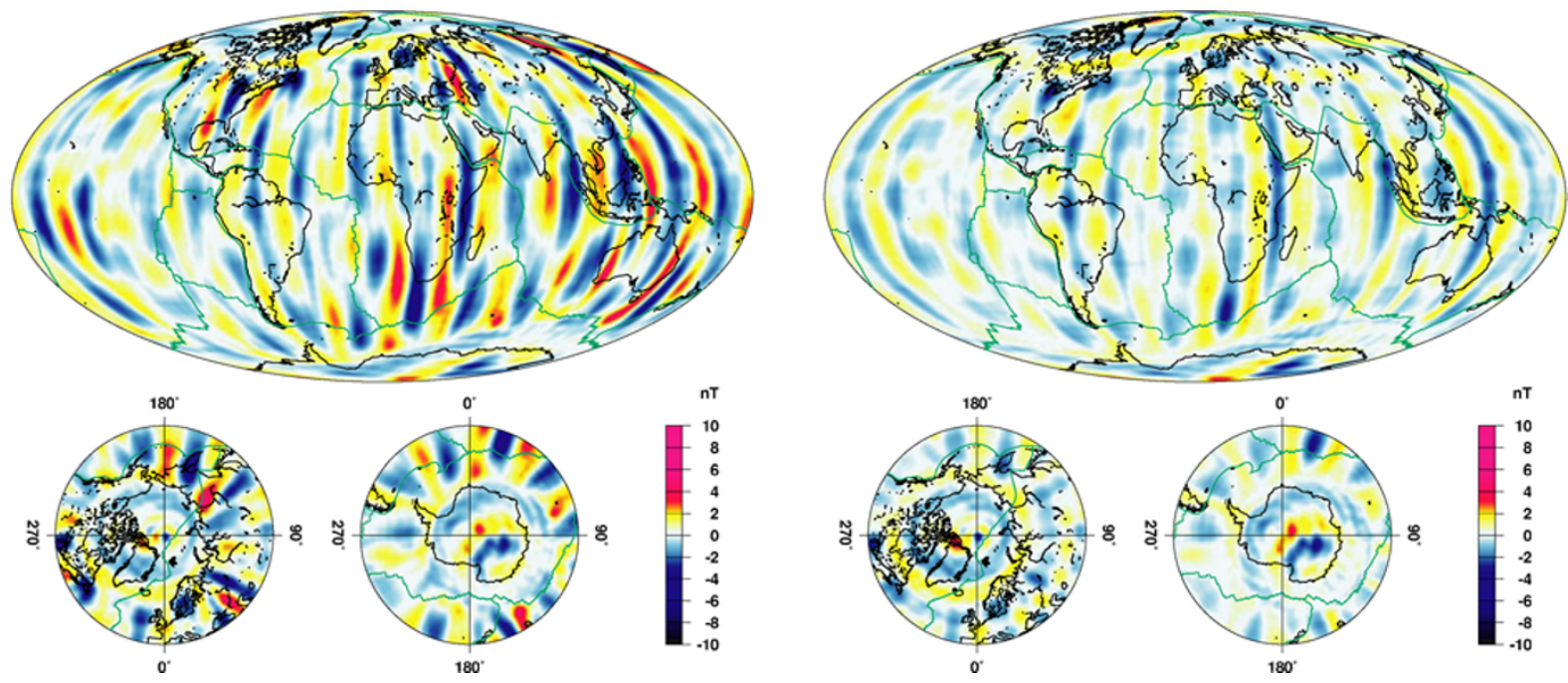

Fig. 8. Global distribution at $400 \mathrm{~km}$ altitude of residuals between input and derived model, (left) solution from satellite C2-S4, (right) solution from satellite pair C2-S4/C2-S5 in Constellation 2.
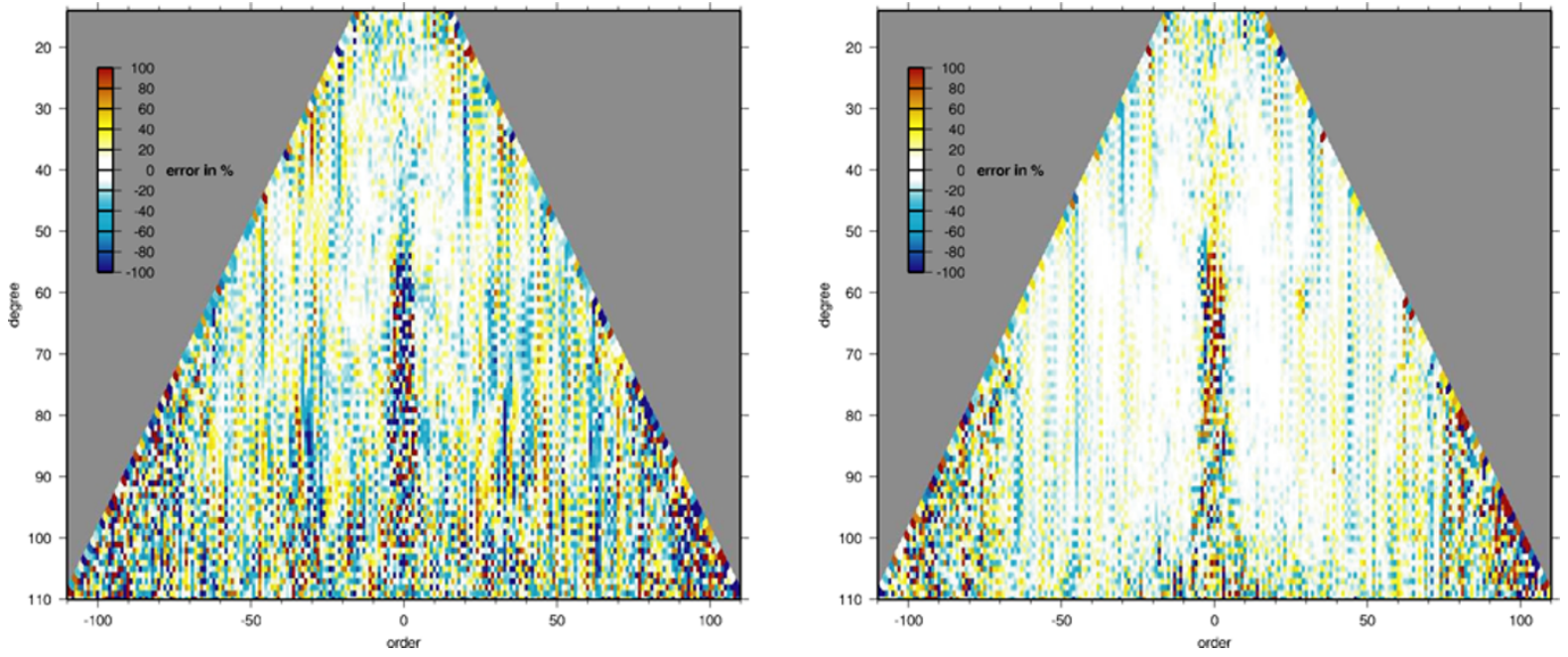

Fig. 9. Sensitivity matrix showing the difference between input and derived SH coefficients as relative error, (left) solution from satellite C2-S4, (right) solution from satellite pair C2-S4/C2-S5 in Constellation 2.

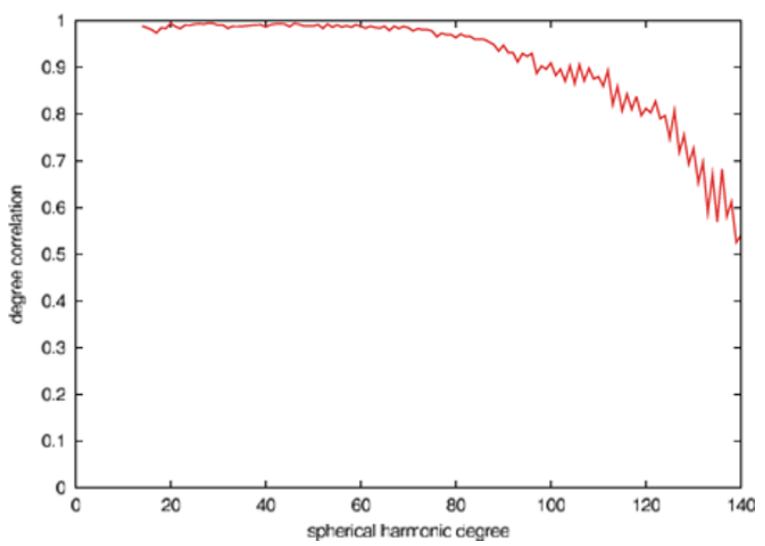

Fig. 10. Degree correlation between input and retrieved lithospheric magnetic field model for the high-degree inversion of C2-S4/C2-S7 in Constellation 2 . data were included in a joint determination of the external contributions.

In the case where two of the lower altitude satellites were used, three types of input data were used in combination: (1) the data given for each satellite, (2) the along-track gradients for each satellite, and (3) the across track gradients between pairs of satellites. These gradients were treated in the least squares inversion in the same manner as the non-gradient vector data. The covariance between the nongradient and gradient data, although the latter are derived from the former, was not taken into account here.

\subsection{Results of the lithospheric field retrieval from Con-} stellation 2

The effects to be investigated in the following simulations included the effect of realistic instrument noise and the spacing of the satellites. The same multi-satellite data set was processed several times using a variety of spacecraft combinations. The most instructive results were obtained from different combinations of the satellites in the lower or- 

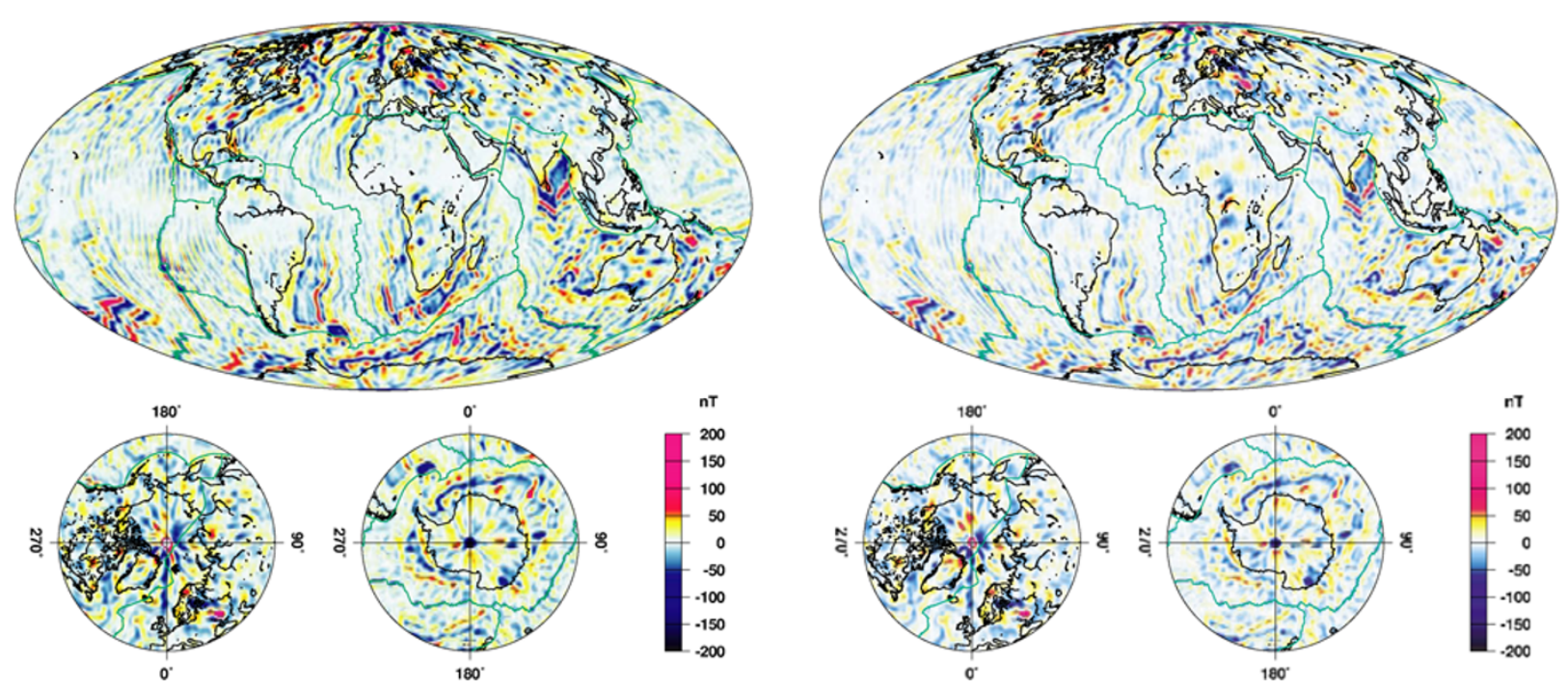

Fig. 11. Global $B_{z}$ map at $50 \mathrm{~km}$ altitude of lithospheric magnetic field. High-resolution (degree and order 150) input model (left), recovered lithospheric features from the C2-S4/C2-S7 pair utilizing the gradient method (right).

bit. Adding one or two spacecraft on a higher orbit did not improve the resolution of the high-degree field.

For the lithospheric field recovery the SH coefficients were determined again for degree and order 14 to 110 . These values were then compared with the SH coefficients of the input model. Figure 7 shows the degree of correlation of three modeling results with the true input model. Using only one low-flying satellite (C2-S4) already recovered the lithospheric signal well. This confirms the findings of the Constellation 1 results. Adding another satellite significantly improved the model accuracy in the longer wavelength range up to degree 30 . The overall gain when considering a second satellite depends quite significantly on the chosen separation. The pair C2-S4/C2-S6 with an E/W separation of $11.25^{\circ}$ in longitude provided good performance up to about $n=30$ where the correlation drops off. Around this degree the wavelength of the signal becomes comparable to the spacecraft separation. For degrees beyond $n=40$ the pair C2-S4/C2-S6 did not provide any advantage over the single-satellite solution.

There is a large difference in the case of the closely spaced pair C2-S4/C2-S5 which is separated only by $1.5^{\circ}$ in longitude (Fig. 7). Here we obtained a significant gain in accuracy over the whole spectrum. The improvement even increases toward higher degrees. At $n=110$ the correlation between the input and the retrieved model is still above 0.9. In the subsequent assessment we therefore compare only the result of C2-S4 with the pair C2-S4/C2-S5.

Figure 8 shows the results from the single satellite and the closely spaced pair C2-S4/C2-S5 models. The dominant features are sectorial stripes. Their spatial scale is close to the truncation wavelength at degree 14 . In the case of the dual-satellite solution the amplitude of the residuals is significantly reduced.

In Figure 9 the relative error of each $\mathrm{SH}$ coefficient is plotted. Evidently, the closely spaced pair provided better results at all degrees. Remaining uncertainties are centred around some $m$ values, concentrated for the C2-S4/C2-S5 pair around $m=0$ and $|m| \sim n$, with the larger errors

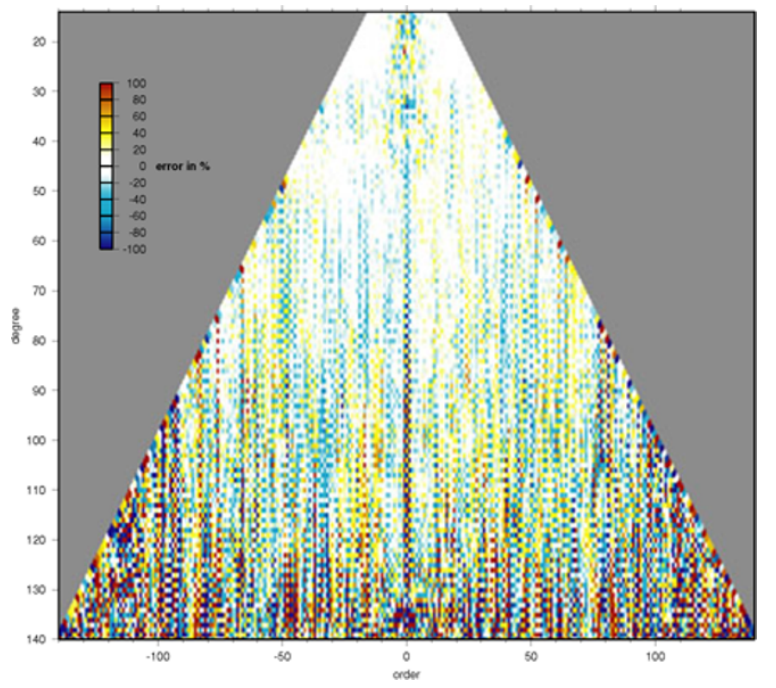

Fig. 12. Sensitivity matrix showing the difference between input and derived $\mathrm{SH}$ coefficients as relative error for the high degree inversion of C2-S4/C2-S7 data in Constellation 2.

around $m=0$ probably caused by the polar gap.

\subsection{Discussion of Constellation 2 results and a new high-degree test}

The Constellation 2 simulation of the recovery of the lithospheric field was based on realistic data even including instrument noise (Olsen et al., 2006). The quality of the retrieved models is remarkably high. It was demonstrated that features up to degree 110 can be recovered reliably with an optimised constellation, comprising spacecraft flying sideby-side. When comparing it with the Constellation 1 analysis, the omission of the unrealistic toriodal field contribution made a big difference.

The key to making optimum use of the side-by-side flying satellites is to use the horizontal gradients of the magnetic field vector measurements. This strongly amplifies the signal to noise ratio. The separation of the spacecraft at the equator should be of the order of half the wavelength of 
the highest degree to be recovered. This is of importance for the design of the constellation. If we aim in the Swarm mission at a model resolution of degree and order $n$, a separation of about $\pi R_{E} / n$ would be optimal. For a goal of degree $n=150$ a separation of $130 \mathrm{~km}$ should then be selected. The observational requirement on the constellation resulting from the lithospheric field objective thus would be to have two spacecraft flying side-by-side separated in the $\mathrm{E} / \mathrm{W}$ direction by about $1.5^{\circ}$ in longitude at the equator crossing.

Motivated by the very promising result of the above study we decided to carry this gradient approach a step further. A higher degree lithospheric model was synthesised from a magnetisation model of the Earth's crust (Olsen et al., 2006). Magnetic field readings were sampled at $5 \mathrm{~s}$ intervals along the orbits of the lower pair, C2-S4 and C2-S7. The orbit of C2-S7 was actively maintained at a distance $1.5^{\circ}$ in longitude east of $\mathrm{C} 2-\mathrm{S} 4$ at the equator. This separation was seen as optimal for a high-degree field recovery.

We aimed at recovering the lithospheric signal to degree and order 140. For this exercise, all magnetic field contributions in the synthetic data set were considered, except for the toroidal field (cf. comments in Section 4.1). The first step was, as usual, the separation of the external field contributions. In a joint inversion, the $\mathrm{SH}$ coefficients of the first three $\mathrm{SH}$ degrees of the internal and external contributions were determined from the readings of C2-S4 and C2S7 on a track-by-track basis and subsequently subtracted. The full vector information of the cleaned data was used to derive the horizontal gradients along track, and acrosstrack between the spacecraft $\mathrm{C} 2-\mathrm{S} 4$ and $\mathrm{C} 2-\mathrm{S} 7$. The data were counted in equal area bins and downweighted by their number density to obtain an equal area distribution on the sphere. In a first inversion, the raw distribution of SH coefficients was estimated. From that an empirical damping matrix was constructed reducing the power of all coefficients which exceeded the average value of the coefficients in their vicinity (in terms of the $n$ by $m$ map of coefficients) by more than $200 \%$. In a second inversion the final SH coefficients were determined by a damped inversion using the empirical damping matrix. In order to check the quality of the results, a degree correlation with the input model was performed. From Figure 10 it can be seen that the agreement is good (close to 1) over a wide range of degrees. The correlation does not drop below 0.7 until degree 130. This is an excellent confirmation of the potential of the field gradient method. A better impression of the extent to which crustal detail is recovered can be obtained from Figure 11 showing the input model on the left and the retrieved map on the right hand side, both at $50 \mathrm{~km}$ altitude. All of the details, especially the ocean bottom stripes, come out very clearly. Figure 12 again shows the relative errors as a sensitivity matrix ordered by degree $n$ versus order $m$. Apart from the polar gap problem for $m=0$, coefficient errors are quite randomly distributed. Thus, there is no indication of a particular group of coefficients being poorly resolved. This is a major improvement over the inversions of single satellite CHAMP data, where the high order $(|m|$ close to $n$ ) coefficients tend to "blow up" (Maus et al., 2006). The E/W gradients of the lower Swarm pair will provide exactly this missing information.

This high-degree additional study demonstrated the large potential for detailed and accurate lithospheric field recovery when dedicated satellite constellations are available. Employing the field gradients from an optimally spaced pair of satellites is the key factor in its success. The demand on computing power is quite large for such a high-degree solution. To assemble the normal equations matrix for the degree and order 140 model required 3 weeks of system time on a 8 processor SUN Fire V880. Once the normal matrix was assembled, the solution of the normal equations took only about 10 minutes.

\section{Conclusions}

We have presented in this paper several tests of recovering crustal magnetic signatures. The analysis is based on near-realistic synthetic data sets. Special emphasis was placed on reliable retrieval of the short wavelength features.

To determine the most suitable measurement configuration in a multi-satellite mission, several runs with different constellations were carried out. The most promising results were obtained from low-flying pairs of satellites which are separated in the E/W direction by 100 to $200 \mathrm{~km}$. Employing the horizontal gradients of the magnetic field vectors significantly improves the ability to resolve small-scale features. To take full advantage of the field gradient information, the separation between the side-by-side flying spacecraft should be comparable to half of the smallest wavelength to be resolved. However, in case of too small a separation, the gradient estimates may degrade due to the noise contribution in the individual readings. This anticipated degradation in the quality of gradients at very small separations could be investigated in a further study prior to launch.

Based on the results of the end-to-end mission performance simulator study it was decided that the Swarm mission shall comprise three spacecraft, one satellite in a higher orbit and a pair flying side-by-side in a lower orbit. This lower pair will provide the relevant data for the high-resolution field models. The anticipated separation is $1.4^{\circ} \pm 0.2^{\circ}$ in longitude. It may be advisable to start with a slightly larger separation and reduce it when the orbit reaches a lower altitude, in order to maintain a constant signal to noise ratio in the gradients.

Acknowledgments. The Swarm End-to-End Mission Performance Simulator Study was funded by ESA through contract No 17263/03/NL/CB. The residual maps and sensitivity matrices were plotted using GMT (Wessel and Smith, 1991).

\section{References}

Arkani-Hamed, J., R. A. Langel, and M. Purucker, Scalar magnetic anomaly maps of Earth derived from POGO and Magsat data, J. Geophys. Res., 99, 24,075-24,090, 1994.

Backus, G., R. L. Parker, and C. Constable, Foundations of Geomagnetism, Cambridge Univ. Press, 1996.

Cohen, Y and J. Achache, New global vector magnetic anomaly maps derived from Magsat data, J. Geophys. Res., 95, 10,783-10,800, 1990.

Friis-Christensen, E., H. Lühr, and G. Hulot, Swarm: A constellation to study the Earth's magnetic field, Earth Planets Space, 58, this issue, 351-358, 2006.

Langel, R. A. and W. J. Hinze, The Magnetic Field of the Earth's Lithosphere-The Satellite Perspective, Cambridge Univ. Press, 1998. 
Maus, S., M. Rother, R. Holme, H. Lühr, N. Olsen, and V. Haak, First scalar magnetic anomaly map from CHAMP satellite data indicates weak lithospheric field, Geophys. Res. Lett., 29(14), 10.1029/ 2001GL013685, 2002.

Maus, S., M. Rother, K. Hemant, C. Stolle, H. Lühr, A. Kuvshinov, and N. Olsen, Earth's lithospheric magnetic field determined to spherical harmonic degree 90 from CHAMP satellite measurements, Geophys. J. Int., 164, 319-330, doi:10.1111/j.1365-246X.2005.02833.x, 2006

Olsen, N., R. Haagmans, T. J. Sabaka, A. Kuvshinov, S. Maus, M. E. Purucker, M. Rother, V. Lesur, and M. Mandea, The Swarm End-to-End mission simulator study: A demonstration of separating the various contributions to Earth's magnetic field using synthetic data, Earth Planets Space, 58, this issue, 359-370, 2006.
Ravat, D., R. A. Langel, M. Purucker, J. Arkani-Hamed, and D. E. Alsdorf, Global vector and scalar Magsat magnetic anomaly maps, J. Geophys. Res., 100, 20,111-20,136, 1995.

Regan, R. D., J. C. Cain, and W. M. Davis, A global magnetic anomaly map, J. Geophys. Res., 80, 794-802, 1975.

Tapley, B. D., S. Bettadpur, M. Watkins, and C. Reigber, The gravity recovery and climate experiment: Mission overview and early results, Geophys. Res. Lett., 31, 10.1029/2004GL019920, 2004.

Wessel, P. and W. H. F. Smith, Free software helps map and display data, EOS Trans. AGU, 72, 441, 1991.

S. Maus (e-mail: Stefan.Maus@noaa.gov), H. Lühr, and M. Purucker 